\title{
ENSAYOS PRELIMINARES EN LA SÍNTESIS VERDE DE NANOPARTICULAS DE PLATA CON EXTRACTOS DE YERBA MATE (Ilex paraguarienis)
}

Data de aceite: 01/08/2020

Data de submissão: 09/05/2020

Mónica Mariela Covinich

Facultad de Ciencias Exactas, Químicas y Naturales, Universidad Nacional de Misiones Félix de Azara 1551 - Posadas - Misiones -

Argentina

Griselda Patricia Scipioni

Facultad de Ciencias Exactas, Químicas y Naturales, Universidad Nacional de Misiones Félix de Azara 1551 - Posadas - Misiones Argentina

\section{David Leopoldo Brusilovsky}

Facultad de Ciencias Exactas, Químicas y Naturales, Universidad Nacional de Misiones Instituto de Materiales de Misiones, CONICET - UNAM

Félix de Azara 1551 - Posadas - Misiones Argentina

RESUMEN: El objetivo del presente trabajo fue ensayar la capacidad reductora de extractos de hojas y palos de yerba mate en la síntesis de nanopartículas de plata (AgNPs). Las muestras de palos y hojas se obtuvieron a partir de yerba mate canchada. La bio-reducción de los iones plata fue monitoreada por espectroscopia UVVisible en el medio de reacción original y en las AgNPs parcialmente purificadas, en un rango de longitudes de onda desde 350 a $600 \mathrm{~nm}$. Todas las experiencias se realizaron al abrigo de la luz. El registro de los espectros mostró un amplio pico de absorción a una longitud de onda aproximada de $450 \mathrm{~nm}$ que se desplazó ligeramente en función del tiempo de reacción y de la concentración del extracto. Los cambios de color del medio de reacción fueron similares a los descriptos en bibliografía para la biosíntesis de AgNPs. Los resultados obtenidos indican que la longitud de onda de máxima absorción y su desplazamiento en función del tiempo de reacción se pueden asignar a la resonancia plasmónica de las AgNPs y que una menor concentración de principios activos en los palos causa las diferencias en los valores de absorbancia observados. La caracterización de las AgNPs fue realizada por microscopía electrónica de barrido (SEM) para confirmar su tamaño nanométrico.

PALABRAS CLAVE: Nanopartículas, Plata, Síntesis, Extractos, Yerba-mate.

\section{PRELIMINARY TESTS ON THE GREEN SYNTHESIS OF SILVER NANOPARTICLES WITH YERBA MATE EXTRACTS (Ilex paraguariensis)}

ABSTRACT: The objective of the present work was to test the reducing capacity of extracts of yerba mate leaves and sticks in the synthesis of silver nanoparticles (AgNPs). The stick and leaf samples were obtained from dried minced yerba mate. The bio-reduction of silver ions was monitored by UV-Visible spectroscopy in the original medium and in the partially purified AgNPs, in a wavelength range from 350 to 600 $\mathrm{nm}$. All experiences were carried out protected from light. The recording of the spectra showed a broad absorption peak at a wavelength of approximately $450 \mathrm{~nm}$, which shifted slightly depending on the reaction time and the concentration of the extract. The changes in the color of the reaction medium were similar to those described in the literature for AgNPs 
biosynthesis. The results obtained indicate that the wavelength of maximum absorption and its displacement as a function of reaction time can be assigned to the plasmonic resonance of the AgNPs and that a lower concentration of active principles in the sticks causes the differences in the observed absorbance values. To confirm the nanometric size of the AgNPs, the characterization of synthetized particles was carried out by scanning electron microscopy (SEM).

KEYWORDS: Nanoparticles, Silver, Synthesis, Extracts, Yerba-mate.

\section{1| INTRODUCCIÓN}

El desarrollo de la nanotecnología se ha convertido en un precursor importante para la exploración científica en física, química e ingeniería. En este contexto, las nanopartículas inorgánicas presentan algunas propiedades electrónicas, ópticas y magnéticas que son diferentes a las características de los mismos compuestos en su fase a granel (Maestro 2012, Bayda 2020). Estas características están directamente relacionadas con el tamaño y la forma de las partículas (Pileni, 2007). Recientemente, se han encontrado varias aplicaciones para nanopartículas metálicas en áreas tales como biología, agricultura, alimentos (Aschberger 2014, Simbine 2019), medicina y ciencias de la salud (Shrivastava, 2009).

Las nanopartículas de plata (AgNPs) en particular, se han utilizado en el diagnóstico médico, sistemas de suministro de fármacos, sanitación, tratamiento del agua y curación de heridas (Chaloupka, 2010). Un estudio sugiere que las nanopartículas de plata actúan disminuyendo la inflamación a través de la modulación de citoquinas (Park, 2011).

Pueden ser sintetizadas por métodos tradicionales o por métodos de síntesis verde. Estos últimos se basan en la biosíntesis reductiva mediante extractos vegetales acuosos y una sal de plata. Numerosos extractos se han utilizado como reactivos reductores, entre ellos pueden citarse los de hojas del árbol de curry (Murraya koenigii) (Qais, 2019), hojas de mangostino (G. Mangostana), hojas de mango (Mangifera índica), frutos de tanaceto (Tansy), jatrofa (Jatropha curcas), hojas del árbol de canela (Cinnamomum verum), hojas de té (Camellia sinensis), Aloe vera y ajo (Allium sativum) (Mittal 2013, Ahmed 2016).

La yerba mate (Ilex paraguariensis) posee una composición química que abarca a varias familias de metabolitos, tales como ácidos orgánicos, lípidos, cafeína, minerales, compuestos fenólicos, azúcares, saponinas y pigmentos (Assis, 2006). Las sustancias fenólicas comprenden flavonoides glicosilados de quercetina y kaenferol, ésteres derivados de los ácidos quínico, cafeico, ferúlico, p-cumárico, y de otros fenoles (Reissman, 1999). La estructura de estos compuestos permite su extracción en solventes polares y les confiere propiedades reductoras que posibilitarían la síntesis de AgNPs (Braganca, 2011). 


\section{I MATERIALES Y MÉTODOS}

\subsection{Materiales}

Las muestras de palos y hojas se obtuvieron a partir de yerba mate (llex paraguariensis) canchada proveniente de una empresa yerbatera ubicada en el sur de la provincia de Misiones, Argentina. Luego de su separación en hojas y palos, cada fracción fue molida y tamizada para eliminar el material con tamaño menor a $500 \mu$ (polvo). El nitrato de plata $\left(\mathrm{AgNO}_{3}\right)$ utilizado fue de grado analítico provisto por Biopack.

\subsection{Síntesis de las nanopartículas de plata}

Los extractos de hojas y palos de yerba mate (medio reductor) se prepararon por extracción acuosa a una temperatura de $70^{\circ} \mathrm{C}$ durante 20 min a partir del material finamente dividido en una relación 1:10 de sólido a solvente. Una alícuota de cada extracto fue diluida hasta obtener soluciones de concentraciones 2,5\%; $5 \%$ y $10 \%$ v/v. A continuación, a una alícuota de cada extracto se le adicionó un volumen fijo de una solución de nitrato de plata $2 \mathrm{mM}$. Todas las soluciones de los precursores fueron previamente reguladas a $\mathrm{pH}$ neutro. Para cada tiempo de reacción establecido, una alícuota del medio de reacción fue centrifugada a $12000 \mathrm{rpm}$ y el precipitado fue redisuelto en agua desionizada. Todas las experiencias se realizaron al abrigo de la luz.

\subsection{Caracterización}

Las propiedades ópticas de las nanopartículas de plata sintetizadas se analizaron mediante espectroscopía UV-Visible con un espectrofotómetro de doble haz (UV-Vis, Shimadzu 2401 PC) equipado con una lámpara de deuterio y ioduro de tungsteno en el rango de 350 a 600 nm a temperatura ambiente. Los espectros se registraron a los 15; 30; 60; 90 y 120 min de reacción.

La morfología, tamaño y composición de las nanopartículas fueron analizados con un microscopio electrónico de barrido Carl Zeiss Supra 40 equipado con una microsonda de fluorescencia de rayos $\mathrm{X}$ por energía dispersiva (EDX).

\section{3| RESULTADOS Y DISCUSIÓN}

La bio-reducción de los iones plata fue monitoreada por espectroscopia UV-Visible en el medio de reacción original y luego en la solución proveniente de la redisolución del precipitado de las AgNPs, en un rango de longitudes de onda desde 350 a $600 \mathrm{~nm}$ (Hyllested, 2015). El registro de los espectros mostró un amplio pico de absorción a una longitud de onda aproximada de $450 \mathrm{~nm}$, asignado a la presencia de AgNPs (Rolim, 2019). Este valor se desplazó ligeramente en función del tiempo de reacción y de la concentración del extracto. Los valores de absorbancia obtenidos fueron inferiores en los medios de reacción formulados con los extractos obtenidos de palos de yerba mate en comparación con los medios de reacción formulados con los extractos obtenidos de hojas para igual concentración de extracto v/v (Figuras 1 y 2). Los desplazamientos en la longitud de onda luego de 120 minutos de reacción fueron $23 \mathrm{~nm}$ y $13 \mathrm{~nm}$ para las AgNPs sintetizadas con 
extractos de hojas; $21 \mathrm{~nm}$ y $17 \mathrm{~nm}$ para las AgNPs sintetizadas con extractos de palos, a concentraciones del $2,5 \%$ y $5 \% \mathrm{v} / \mathrm{v}$ respectivamente, similares a los informados en otras investigaciones (González, 2104). El comportamiento observado en los extractos al 10\% fue diferente. Los resultados obtenidos indican que la longitud de onda de máxima absorción y su desplazamiento en función del tiempo de reacción se pueden asignar a la resonancia plasmónica de las AgNPs (Slistan-Grijalba 2005, Al-Ghamdi, 2014). Los cambios de color del medio de reacción (y la coloración del precipitado) fueron similares a los descriptos en la bibliografía para la biosíntesis de AgNPs (Falconi 2015, Huang 2010). El color parduzco aparece debido a la oscilación coherente de los electrones de la banda de conducción en la superficie de la AgNPs (Qais, 2019).

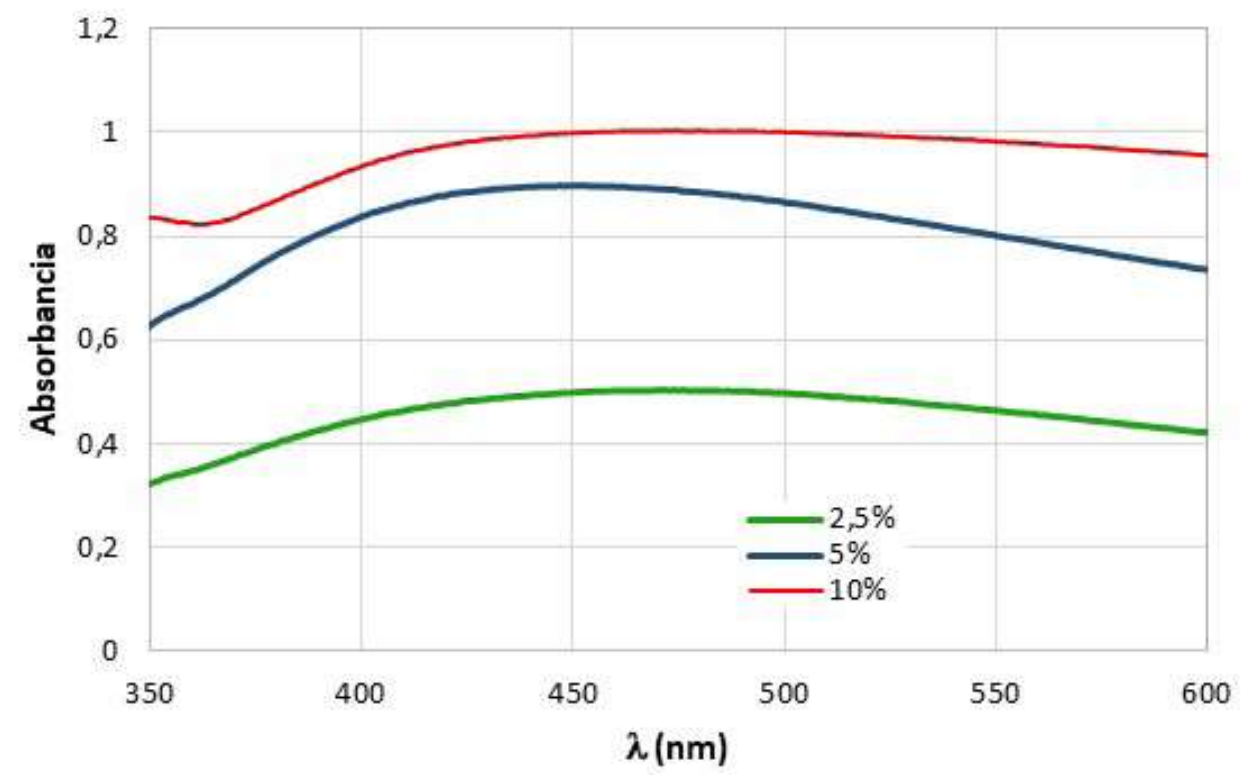

Figura 1. Espectros de absorción de resonancia plasmónica luego de 120 min. de reacción para extractos de hojas de yerba mate. 


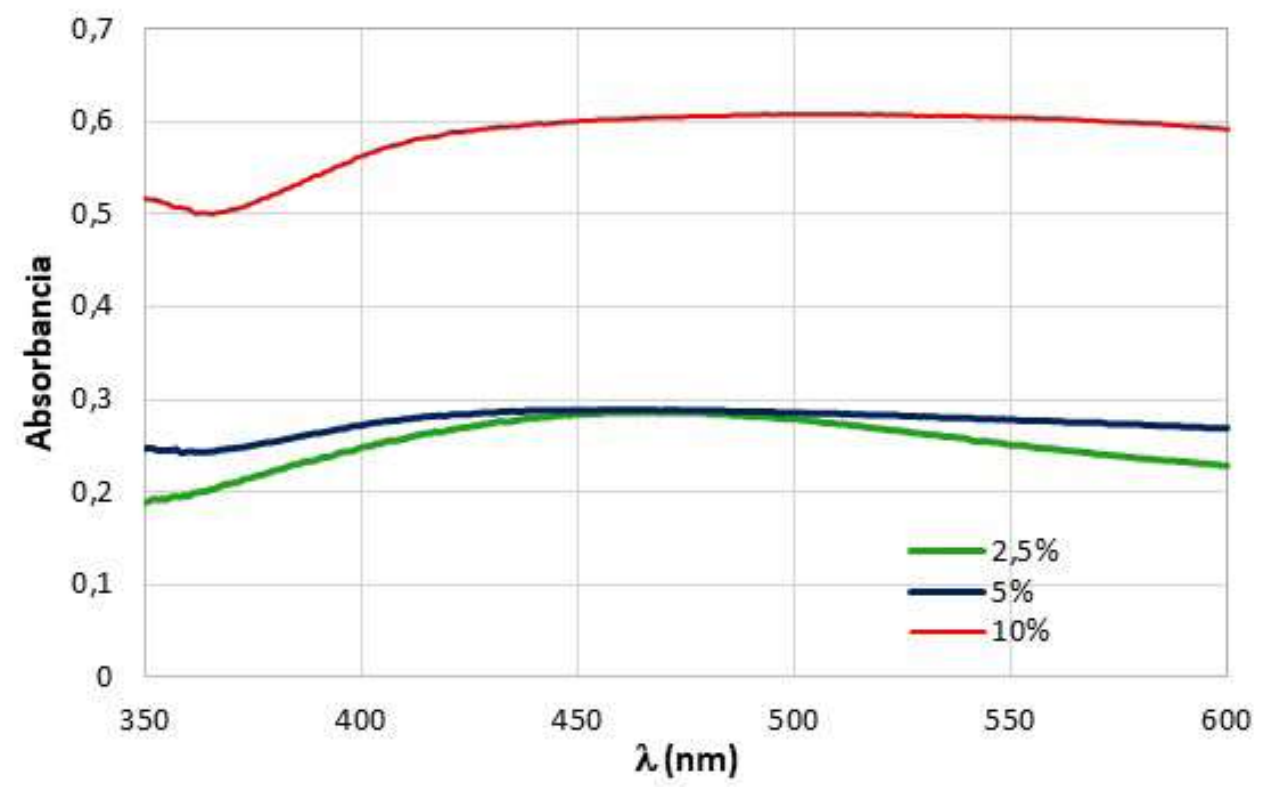

Figura 2. Espectros de absorción de resonancia plasmónica luego de 120 min. de reacción para extractos de hojas de yerba mate.

Una menor concentración de principios activos biorreductores en los medios de reacción preparados con extractos de palos sería la causa de las diferencias en los valores de absorbancia registrados durante la síntesis verde de las AgNPs.

En la Figura 3 puede observarse que las partículas presentaron forma irregular y tamaños variables entre 20 y $60 \mathrm{~nm}$. La dispersión en el tamaño de las AgNPs podría asociarse con el ancho de la banda de máxima Absorbancia obtenida en los espectros de absorción, a diferentes tiempos, durante el progreso de las reacciones de síntesis.
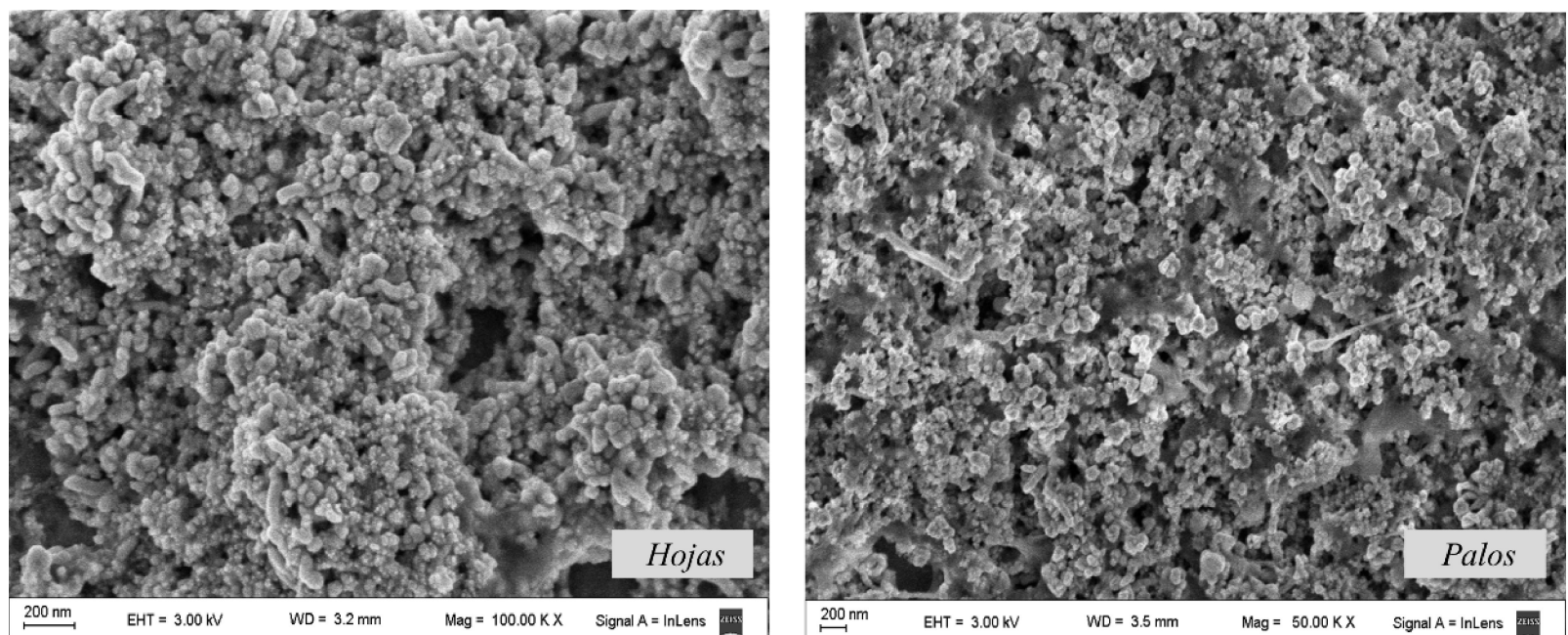

Figura 3. Micrografías de barrido electrónico (SEM) en medio reductor al $5 \%$ y tiempo de reacción de15 minutos 


\section{I CONCLUSIONES}

El extracto de yerba mate es un eficiente medio reductor de iones de plata en la síntesis de nanopartículas. La longitud de onda de máxima absorción y su desplazamiento en función del tiempo de reacción se pueden asignar a la resonancia plasmónica de las AgNPs. Una menor concentración de principios activos reductores en los extractos obtenidos de los palos genera valores de absorbancia menor en relación con los medios de reacción obtenidos a partir de extractos de hojas. La microscopía electrónica de barrido (SEM) confirma la presencia de AgNPs.

\section{REFERENCIAS}

AHMED, S. et al. A review on plants extract mediated synthesis of silver nanoparticles for antimicrobial applications: A green expertise. Journal of Advanced Research, 2016.

AL-GHAMDI, H. S.; MAHMOUD, W. E. Synthesis of self-assembly plasmonic silver nanoparticles with tunable luminescence color. Journal of Luminescence, v. 145, p. 880-883, 2014.

ASCHBERGER, K. et al. EFSA supporting publication 2014. EFSA supporting publication, p. 125, 2014.

ASSIS JACQUES, R. et al. Chemical composition of mate tea leaves (llex paraguariensis): A study of extraction methods. Journal of Separation Science, v. 29, n. 18, p. 2780-2784, 2006.

BAYDA, S. et al. The history of nanoscience and nanotechnology: From chemical-physical applications to nanomedicine. Molecules, v. 25, n. 1, p. 1-15, 2020.

BRAGANÇA, V. L. C.; MELNIKOV, P.; ZANONI, L. Z. Trace elements in different brands of Yerba mate tea. Biological Trace Element Research, v. 144, n. 1-3, p. 1197-1204, 2011.

CHALOUPKA, K.; MALAM, Y.; SEIFALIAN, A. M. Nanosilver as a new generation of nanoproduct in biomedical applications. Trends in Biotechnology, v. 28, n. 11, p. 580-588, 2010.

FALCONÍ, V. E. E. Sintesis y caracterización de nanoparticulas de plata por espectroscopia de infrarrojos (FT-IR), UV-Vis, absorción atómica de llama (FAAS) y microscopía de barrido electrónico (SEM). 2015. PONTIFICIA UNIVERSIDAD CATÓLICA DEL ECUADOR, 2015.

GONZÁLEZ, A. L. et al. Size, shape, stability, and color of plasmonic silver nanoparticles. Journal of Physical Chemistry C, v. 118, n. 17, p. 9128-9136, 2014.

HUANG, T.; XU, X. H. N. Synthesis and characterization of tunable rainbow colored colloidal silver nanoparticles using single-nanoparticle plasmonic microscopy and spectroscopy. Journal of Materials Chemistry, v. 20, n. 44, p. 9867-9876, 2010.

HYLLESTED, J. A. et al. Green preparation and spectroscopic characterization of plasmonic silver nanoparticles using fruits as reducing agents. Beilstein Journal of Nanotechnology, v. 6, n. 1, p. 293299, 2015. 
MAESTRO, J. M. A.; CARBAJO, J. I. M. Aplicaciones Industriales de la Nanotecnología.

Universidad de Oviedo, 2012.

MITTAL, A. K.; CHISTI, Y.; BANERJEE, U. C. Synthesis of metallic nanoparticles using plant extracts. Biotechnology Advances, v. 31, n. 2, p. 346-356, 2013.

PARK, E. J.; CHOI, K.; PARK, K. Induction of inflammatory responses and gene expression by intratracheal instillation of silver nanoparticles in mice. Archives of Pharmacal Research, v. 34, n. 2, p. 299-307, 2011.

PILENI, M. P. Self-assembly of inorganic nanocrystals: Fabrication and collective intrinsic properties. Accounts of Chemical Research, v. 40, n. 8, p. 685-693, 2007.

QAIS, F. A. et al. Antibacterial effect of silver nanoparticles synthesized using Murraya koenigii (L.) against multidrug-resistant pathogens. Bioinorganic Chemistry and Applications, v. 2019, 2019.

REISSMANN, C. B.; RADOMSKI, M. I.; DE QUADROS, R. M. B. Chemical composition of llex paraguariensis St. Hil. under different management conditions in seven localities of Paraná state.

Brazilian Archives of Biology and Technology, v. 42, n. 2, p. 187-194, 1999.

ROLIM, W. R. et al. Green tea extract mediated biogenic synthesis of silver nanoparticles:

Characterization, cytotoxicity evaluation and antibacterial activity. Applied Surface Science, v. 463, p. 66-74, 2019.

SHRIVASTAVA, S.; DASH, D. Applying Nanotechnology to Human Health: Revolution in Biomedical Sciences. Journal of Nanotechnology, v. 2009, p. 1-14, 2009.

SIMBINE, E. O. et al. Application of silver nanoparticles in food packages: A review. Food Science and Technology, v. 39, n. 4, p. 793-802, 2019.

SLISTAN-GRIJALVA, A. et al. Classical theoretical characterization of the surface plasmon absorption band for silver spherical nanoparticles suspended in water and ethylene glycol. Physica E: Low-

Dimensional Systems and Nanostructures, v. 27, n. 1-2, p. 104-112, 2005. 